\title{
Preparation of Cellulose Particles Using an Ionic
}

\section{Liquid}

Toyoko Suzuki, Kyosuke Kono, Kengo Shimomura, Hideto Minami *

Department of Chemical Science and Engineering, Graduate School of Engineering, Kobe University, Rokko, Nada, Kobe 657-8501, Japan

E-mail: minamihi@kobe-u.ac.jp, TEL \& FAX: (+81) 788036197 


\begin{abstract}
Cellulose is a ubiquitous natural fiber used in various industrial materials and applications. We prepared micron-sized cellulose particles by the solvent releasing method (SRM) in which cellulose-[Bmim]Cl- $N, N$-dimethylformamide (DMF) droplets are dispersed in hexadecane (HD) containing dissolved surfactant. The dispersion is then poured into a large amount of 1-butanol. Since 1-butanol is miscible with $\mathrm{HD},[\mathrm{Bmim}] \mathrm{Cl}$, and $\mathrm{DMF}$ but not with cellulose, the cellulose particles precipitate out. FTIR and ${ }^{1} \mathrm{H}$ NMR analyses confirmed that this technique precipitated cellulose and completely removed $[\mathrm{Bmim}] \mathrm{Cl}$ and $\mathrm{DMF}$ from the cellulose-[Bmim]Cl-DMF droplets. Interestingly, the obtained cellulose particles were almost the same size as the original droplets (cellulose, $7 \mathrm{wt} \%$ ), indicating a microporous structure of the cellulose particles with a large medium content. Although the microporous structure collapsed as the medium evaporated, it was maintained by a freeze-drying technique.
\end{abstract}

\title{
Keywords
}

Colloids, Cellulose, Ionic liquid, Porous structure 


\section{Introduction}

Cellulose is the most abundant natural polymer on Earth; it is an attractive industrial material because it is inexpensive, chemically stable, nontoxic, biodegradable, and modifiable.[1] Cellulose is extensively used in materials such as regenerated fibers, membranes, nonwoven fabrics, and pulp.[2-4] Recently, the utility of cellulose particles as packaging materials for bio-chromatography and as carrier materials for diagnostic reagents has been recognized.[5-9] Cellulose particles are commonly prepared by dropping or spraying cellulose solutions into a coagulation bath $[10,11]$ or dispersing in an immiscible medium. In the latter approach, the dispersion is formed by mechanical stirring with a surfactant, and the solvent is evaporated from the cellulose solution droplets.[12-14] However, solvent dissolution is necessary in multistep processes or drastic conditions such as those occurring in the viscose method for the dissolution of cellulose, since cellulose is intra- and inter-molecularly connected by hydrogen bonds, and are therefore insoluble in water and most organic solvents.[15-17]

Throughout the past decade, ionic liquids (ILs) have become increasingly recognized as environmentally friendly media. Some ILs readily dissolve organic and inorganic materials that are insoluble in most conventional solvents.[18-21] In 2002, Rogers and colleagues[22] reported that ILs such as 1-butyl-3-methylimidazolium chloride 
$([\mathrm{Bmim}] \mathrm{Cl})$ require only mild heat treatment to dissolve cellulose. Thereafter, there has been increasing interest in the dissolution of cellulose using ILs.[23-26] The mixtures of aprotic polar organic solvents such as $N, N$-dimethylformamide (DMF) with small fractions of ILs could instantaneously dissolve the cellulose even at high concentrations at mild conditions.[27, 28] Furthermore, the processing of cellulose using ILs were also reported; for example, preparation of cellulose films[29], fibers[30, 31], and composite materials of cellulose and other polymers.[32-34] The cellulose-[Bmim]Cl solution is highly viscous and leads to spontaneous gelation.[35] Moreover, many researchers have exploited IL solubility to investigate acetylation, acylation and silylation[36-39] of cellulose and also bioethanol production from cellulose.[40] Song and colleagues[8], and independently, Lin and colleagues[41], prepared cellulose particles by cooling an oil-IL solution containing dissolved cellulose suspension and adding water or alcohol to the cooled suspension. However, in these previous studies, the cellulose particles were not characterized in detail, and no attempt was made to control their morphology. Morphological control is essential if cellulose is to be widely adopted in the biomedical fields.

In this study, cellulose particles were obtained and characterized by the solvent releasing method $(\mathrm{SRM})$ conducted at room temperature using cellulose-[Bmim]Cl- 
DMF droplets. The formation mechanism of cellulose particles was also discussed. We originally proposed SRM for preparing spherical or nonspherical polymer particles and composite particles by reconstructing dissolved polymers.[42-44] Briefly, the method involves pouring an aqueous dispersion of toluene droplets containing dissolved polymers into a nonsolvent.

\section{Experimental section}

\subsection{Materials}

Microcrystalline cellulose (powder, derived from cotton linter) and the IL 1-butyl-3-methylimidazolium chloride $([\mathrm{Bmim}] \mathrm{Cl})$ were used as received from Aldrich Chemical Co. Ltd. DMF, n-hexadecane (HD), 1-butanol and acetone were used as received from Nacalai Tesque Inc. (Kyoto, Japan). Sorbitan monooleate (Span 80, Kao Co.; Tokyo, Japan) (RHEODOL SP-O10, HLB 4.3), polysiloxane series surfactant Silaplane FM-3321 (Chisso Co.; Tokyo, Japan), DMF-d7 (Eurisotop; Essonne, France), and 1-butanol- $d_{10}$ (ISOTEC; Ohio, USA) were also used as received. 


\subsection{Preparation of cellulose particles by the SRM}

Cellulose $(15 \mathrm{wt} \%)$ was dissolved in $[\mathrm{Bmim}] \mathrm{Cl}[35]$ by heating at $100^{\circ} \mathrm{C}$ for $8 \mathrm{~h}$ in an oil bath. The resulting cellulose solution was stored in an oven at $80^{\circ} \mathrm{C}$ to avoid moisture.

Cellulose particles were prepared by SRM with cellulose-[Bmim]Cl-DMF droplets.

Since the viscosity of the cellulose- $[\mathrm{Bmim}] \mathrm{Cl}$ solution increases at room temperature, DMF was added as a cosolvent.[30] A solution of cellulose-[Bmim]Cl-DMF (7/43/50, w/w/w) (0.2 g) was mixed with HD (2.0 g) and Span 80 or Silaplane FM-3321 as a stabilizer ( $0.25 \mathrm{wt} \%$ of HD) and stirred at 10,000 rpm for 5 min using a homogenizer (BM-1, Nihonseiki Kaisha Ltd.; Tokyo, Japan). The dispersion was poured into excess 1-butanol (ca. $20 \mathrm{~g}$ ) with magnetic stirring at $200 \mathrm{rpm}$ to remove [Bmim] $\mathrm{Cl}$ and $\mathrm{DMF}$ from the cellulose-[Bmim]Cl-DMF droplets, that is, to precipitate the cellulose particles. The precipitates were washed three times $\left(1^{\text {st }}\right.$ wash; 1 -butanol, $2^{\text {nd }}$ wash; acetone, $3^{\text {rd }}$ wash; acetone) to remove remaining $[\mathrm{Bmim}] \mathrm{Cl}, \mathrm{DMF}$, and HD. In the freeze-drying process, the cellulose particles redispersed in water were immersed in liquid nitrogen for a few minutes and then freeze-dried in a freeze drier (FDU-1200, Tokyo Rikakikai Co.; Ltd., Tokyo, Japan). 


\subsection{Characterization}

Cellulose particles were observed by optical microscopy (ECLIPSE 80i;

Nikon, Tokyo, Japan) and scanning electron microscopy (SEM, JSM-6510, JEOL Ltd.;

Tokyo, Japan). The components of the cellulose particles were analyzed using a Fourier transform infrared spectrometer (FTIR, FT/IR-6200, JASCO Corporation; Tokyo, Japan) using the pressed $\mathrm{KBr}$ pellet technique. The dissolution state of cellulose molecules was confirmed by ${ }^{1} \mathrm{H}$ NMR (Avance 500; Bruker, Massachusetts, USA) measurements of the cellulose-[Bmim]Cl-DMF- $\mathrm{d}_{7}$ solution and 1-butanol- $\mathrm{d}_{10}$. Following SRM, the transmittance of the cellulose particles redispersed in water was measured at $200 \mathrm{~nm}-800 \mathrm{~nm}$ using a UV-vis spectrophotometer (UV-2500, Shimadzu Corporation; Kyoto, Japan). The specific surface area of the freeze-dried cellulose particles was analyzed using a surface area analyzer (NOVA 3200e, Quantachrome Instruments; Florida, USA) using nitrogen as sorption gas. 


\section{Result and discussion}

\subsection{Preparation of cellulose particles by the SRM}

Figure 1a shows a dispersion of cellulose-[Bmim]Cl-DMF droplets in HD using polysiloxane (Silaplane FM-3321) as a high-molecular-weight colloidal stabilizer, which retained the colloidal stability of the droplets for a few minutes. In the presence of lower-molecular-weight stabilizers such as Span 80 and Span 85, the droplets had coalesced within several seconds. SRM and washing processes of the Silaplane FM-3321 system yielded spherical cellulose particles whose colloidal stability was maintained. Throughout the washing process, the cellulose particles remained noncoalescent, and their morphologies appeared unaltered. The optical micrograph showed no obvious contrast between the particles (refractive index of 1.50) and medium (1-butanol and acetone; refractive indexes of 1.40 and 1.36, respectively). The cellulose particles retained low contrast, even when acetone was replaced with water. Moreover, the dispersion of cellulose particles following SRM was visually transparent (see Figure 1e, inset at upper right). These observations suggest that the obtained cellulose particles are swollen with $[\mathrm{Bmim}] \mathrm{Cl}$ (a strong cellulose solvent), which likely remained in the particles after SRM and washing processes. 


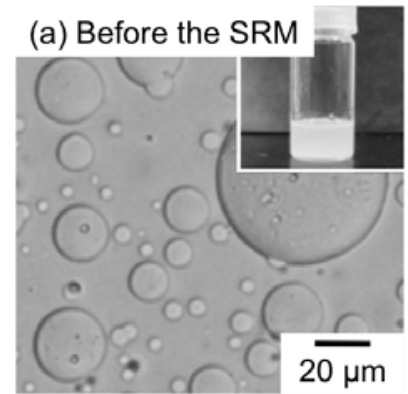

(b) After the SRM

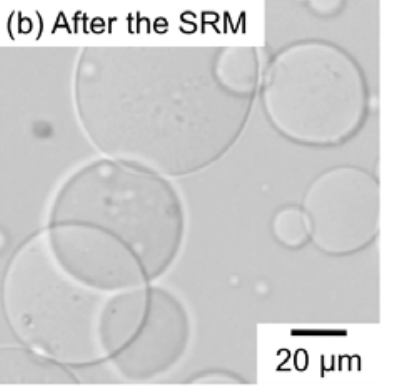

(c) $1^{\text {st }}$ wash (1-butanol)

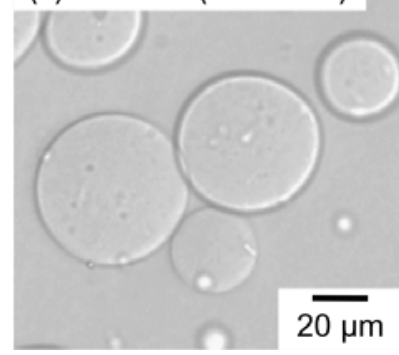

(d) $2^{\text {nd }}$ wash (acetone)

(e) $3^{\text {rd }}$ wash (acetone)
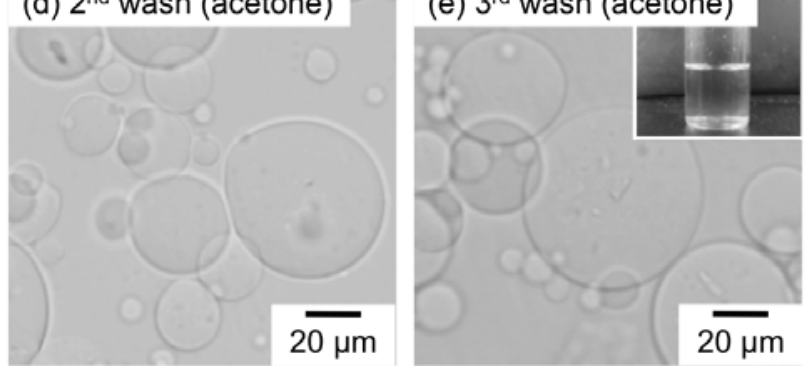

Figure 1. Optical micrographs of cellulose particles before (a) and after (b) the SRM of cellulose-[Bmim]Cl-DMF (7/43/50, w/w/w) droplets dispersed in HD with Silaplane FM-3321 using 1-butanol and after the washing processes (c-e). The insets in (a) and (e) show visual appearances of their dispersions. Washing process: (c) $1^{\text {st }}$ wash (1-butanol); (d) $2^{\text {nd }}$ wash (acetone); (e) $3^{\text {rd }}$ wash (acetone).

Figure 2 shows the FTIR spectra of cellulose particles at each washing step.

For comparison, the spectrum of pure $[\mathrm{Bmim}] \mathrm{Cl}$ and cellulose are also shown. Following SRM, the characteristic peak of $[\mathrm{Bmim}] \mathrm{Cl}$ appeared at around $1600 \mathrm{~cm}^{-1}$ (Figure $2 \mathrm{a}$ ), implying that $[\mathrm{Bmim}] \mathrm{Cl}$ is retained after SRM. This result is expected, since $[\mathrm{Bmim}] \mathrm{Cl}$ should not evaporate during the drying process. However, the peak 
disappeared after the $1^{\text {st }}$ wash, and the spectrum of the washed particles was consistent with that of pure cellulose, indicating that the washing process flushes $[\mathrm{Bmim}] \mathrm{Cl}$ from the cellulose particles. In addition, the characteristic peaks (800 and $1260 \mathrm{~cm}^{-1}$ ) of Silaplane FM-3321 (surfactant) were not observed at all steps, showing that the surfactant also should be removed.

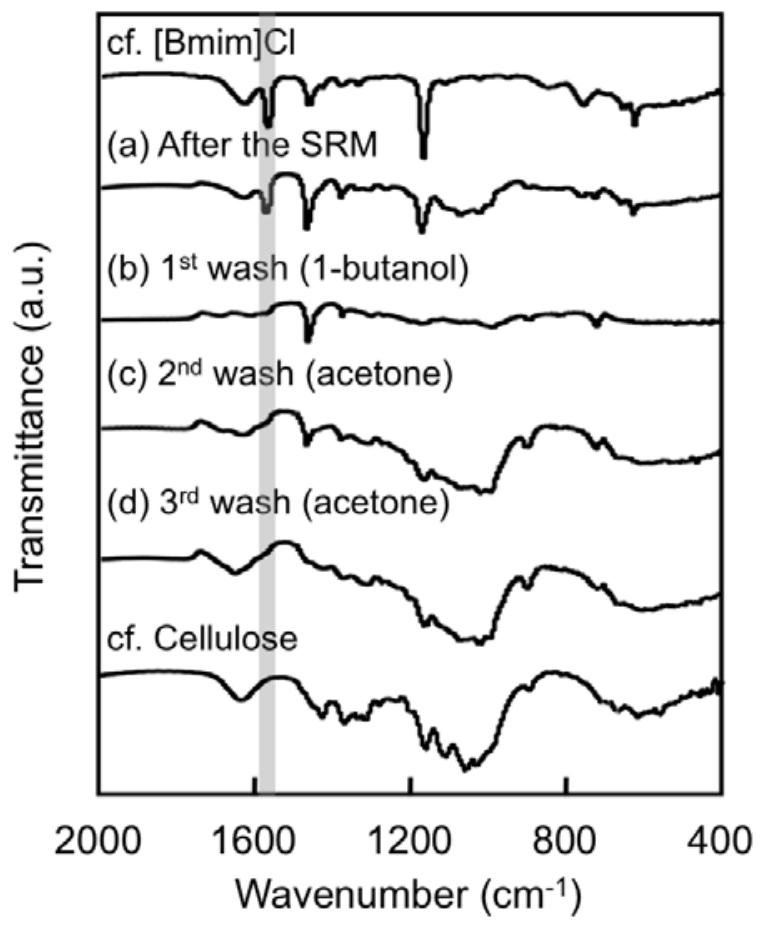

Figure 2. FTIR spectra of cellulose particles after the washing process, prepared by SRM of cellulose-[Bmim]Cl-DMF $(7 / 43 / 50, \mathrm{w} / \mathrm{w} / \mathrm{w})$ droplets dispersed in HD with Silaplane FM-3321 using 1-butanol. Washing process: (a) cellulose particles fabricated by SRM; (b) $1^{\text {st }}$ wash (1-butanol); (c) $2^{\text {nd }}$ wash (acetone); (d) $3^{\text {rd }}$ wash (acetone). 
Figure 3 shows the ${ }^{1} \mathrm{H}$ NMR spectra of cellulose-[Bmim]Cl-DMF- $d 7$ (7/43/50, w/w/w) solution before and after pouring into 1-butanol-d $d_{10}$. To confirm the precipitation of cellulose by SRM, an SRM-like procedure was performed in a deuterated solvent. Millimeter-sized particles were immediately prepared by dropping cellulose- $[\mathrm{Bmim}] \mathrm{Cl}-\mathrm{DMF}-d_{7}$ solution into 1 -butanol- $d_{10}$. Before pouring into 1-butanol-d $d_{10}$, cellulose-derived peaks appeared around 3-4 ppm, as shown in Figure 3a, indicating that cellulose has dissolved in [Bmim]Cl-DMF-d7.[45, 46] After pouring the cellulose solution into 1-butanol- $d_{10}$, these peaks disappeared. Solution-state ${ }^{1} \mathrm{H}$ NMR generally detects only dissolved samples; it is unsuitable for precipitated samples. This result indicates that cellulose dissolved in $[\mathrm{Bmim}] \mathrm{Cl}$ and $\mathrm{DMF}$ is completely precipitated in 1-butanol. Furthermore, the precipitated millimeter-sized particle could be handled by tweezers without deformation, as shown in Figure 4. This strongly supports that SRM-fabricated cellulose particles actually precipitated and that they do not exist in the swelling state. 

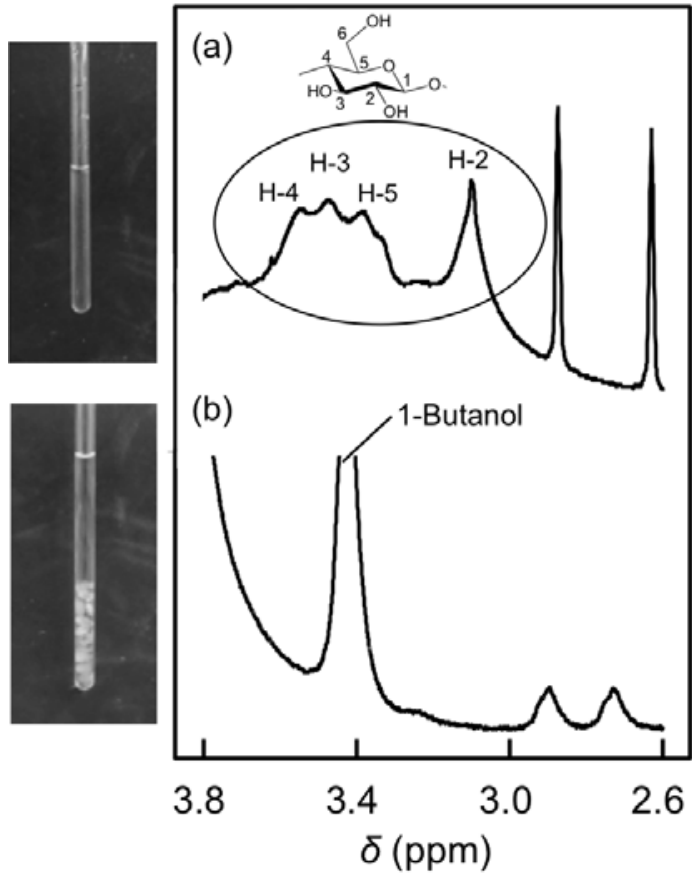

Figure 3. ${ }^{1} \mathrm{H}$ NMR graphs of cellulose-[Bmim]Cl-DMF- $d_{7}(7 / 43 / 50, w / w / w)$ solution before (a) and after (b) pouring into 1-butanol- $d_{10}$

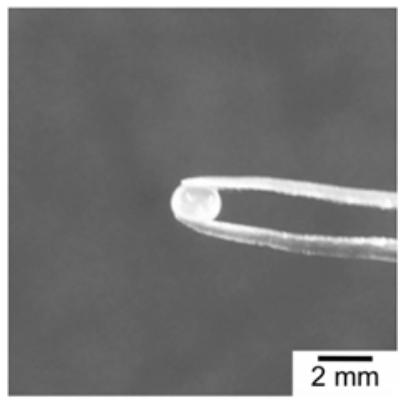

Figure 4. Visual appearance of a cellulose sphere prepared by dropping cellulose-

[Bmim]Cl-DMF (7/43/50, w/w/w) solution into 1-butanol and washing in acetone 


\subsection{Formation mechanism of cellulose particles by the SRM}

To clarify the formation mechanism of cellulose particles, the SRM process of cellulose-[Bmim]Cl-DMF droplets was observed under an optical microscope. Figure 5 shows consecutive optical micrographs of a cellulose-[Bmim]Cl-DMF (7/43/50, w/w/w) droplet dispersed in a solution of HD with Silaplane FM-3321 before, immediately following, and ca. $30 \mathrm{~s}$ after injection of 1-butanol from the edge of a cover glass. Once 1-butanol had diffused to the observing droplet, [Bmim]Cl and DMF were released (Figure 5b). Thirty seconds later, [Bmim] Cl and DMF should have been completely released; however, no morphology changes were observed in the particles at this time (Figure 5c). Notably, the diameter of the droplets was unaltered by the SRM process, although the cellulose content was $7 \mathrm{wt} \%$. Moreover, the diameter and morphology of the wet particles remained unchanged when the medium (1-butanol) was replaced with acetone (wet sample). 

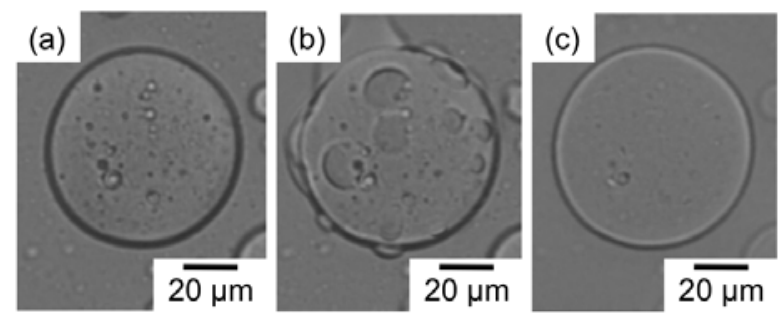

Figure 5. Consecutive optical micrographs of cellulose-[Bmim]Cl-DMF (7/43/50, w/w/w) droplets dispersed in HD with Silaplane FM-3321 as a stabilizer, prepared by a homogenizer before (a), immediately after (b), and ca. $30 \mathrm{~s}$ after (c) addition of 1-butanol

However, the cellulose particles simultaneously shrank as acetone evaporated (Figure 6). The shrunken cellulose particles (dried sample) were almost spherical, and their volumes were $7 \mathrm{vol} \%$ relative to those of wet particles. The percentage of shrinking favorably agreed with the volume fraction of cellulose in the primary droplet. These results suggest that the cellulose particles formed by SRM possess a fragile hollow or porous structure before drying. 


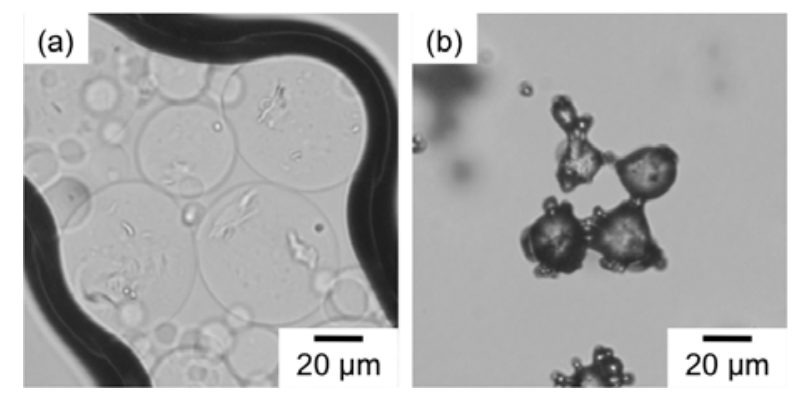

Figure 6. Optical micrographs of cellulose particles dispersed in acetone before (a) and immediately after (b) evaporation of acetone. The particles were prepared by SRM of cellulose-[Bmim]Cl-DMF droplets dispersed in HD with FM-3321 using 1-butanol.

The specific structure of the cellulose particles dispersed in water was investigated by UV-vis analysis (Figure 7). The wet sample was transferred from acetone to water without drying. The dried sample and microcrystalline cellulose were redispersed in water. As shown in Figure 7c, microcrystalline cellulose did not absorb at ultraviolet wavelengths. While the dried sample similarly showed no peak at these wavelengths, a peak was observed around $260 \mathrm{~nm}$ in the spectrum of the wet sample. Thus, the broad peak observed in the spectrum of wet cellulose particles is not attributable to absorption by cellulose or water; however, it arises from scattering by the structure of cellulose 
particles. This observation indicates a nanoscale structure of cellulose particles.[47]

However, the nanoscale structure is fragile, and it collapses under capillary force as the medium evaporates.

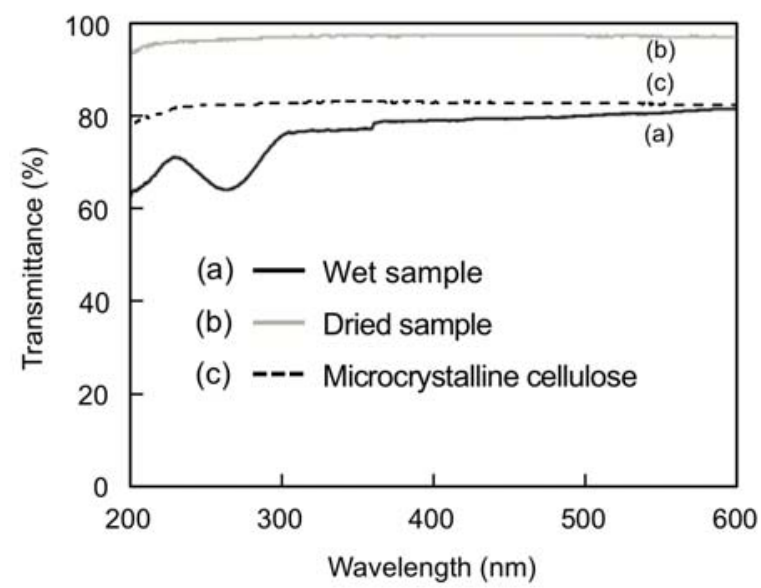

Figure 7. UV-vis spectra of aqueous dispersions of microcrystalline cellulose (c, black dashed line), cellulose particles prepared by SRM using 1-butanol followed by washing with 1-butanol, acetone and water (a, black solid line), and after drying and redispersing in water (b, gray solid line)

Figure 8a, b show SEM photographs of the dried sample and its inner morphology (revealed by fractioning with a razor blade), respectively. The obtained particles had a smooth surface and dense structure, probably resulting from structural collapse accompanying medium evaporation. To prevent such collapse, the obtained particles were dried by a freeze-drying technique. The structure of the freeze-dried particles was 
spongy, revealing several 10 -nm-sized cellulose fibers, although these had been deformed by the pressure of ice crystal growth (Figures $8 \mathrm{c}, \mathrm{d}$ ). The specific surface area of the freeze-dried cellulose particles $\left(44.6 \mathrm{~m}^{2} / \mathrm{g}\right)$, measured by Brunauer-EmmettTeller, exceeded that of dense particles by more than 100 times. The calculated surface area of $10-\mu \mathrm{m}$-sized dense cellulose particles is $0.38 \mathrm{~m}^{2} / \mathrm{g}$ (given that cellulose density $=$ $1.6 \mathrm{~g} \mathrm{~cm}^{-3}$ ). To investigate the influence of cellulose concentration to the particle morphology, the same procedures were carried out in the case of lower cellulose concentration systems. In the cellulose-[Bmim]Cl-DMF $(3 / 47 / 50, \mathrm{w} / \mathrm{w} / \mathrm{w})$ droplets, obtained cellulose particles (after freeze-dry) had a similar morphology, but the porosity seemed to be more sparse. This result indicates that morphology of cellulose particles would be controlled by changing initial cellulose concentration. On the other hand, in the cellulose-[Bmim]Cl-DMF $(1 / 49 / 50, \mathrm{w} / \mathrm{w} / \mathrm{w})$ droplets, some obtained particles were deformed to non-spherical shape, in which cellulose network could not be formed due to exceptionally low cellulose concentration.

From the above findings, we propose the following formation mechanism of cellulose particles by SRM. When cellulose-[Bmim]Cl-DMF droplets dispersed in HD with Silaplane FM-3321 as a stabilizer are poured into excess 1-butanol, HD, [Bmim]Cl, and DMF are immediately mixed with 1-butanol; that is, $[\mathrm{Bmim}] \mathrm{Cl}$ and $\mathrm{DMF}$ in the droplets 
are immediately replaced by 1-butanol. Simultaneously, cellulose, which cannot dissolve in 1-butanol, is precipitated from the droplet surface. This mechanism explains why the cellulose particles are spongy in nature and why their size is unaltered from that of the original droplets. The spongy structure of the cellulose particles is fragile and collapses under capillary force during the drying process, thereby forming dense cellulose particles.
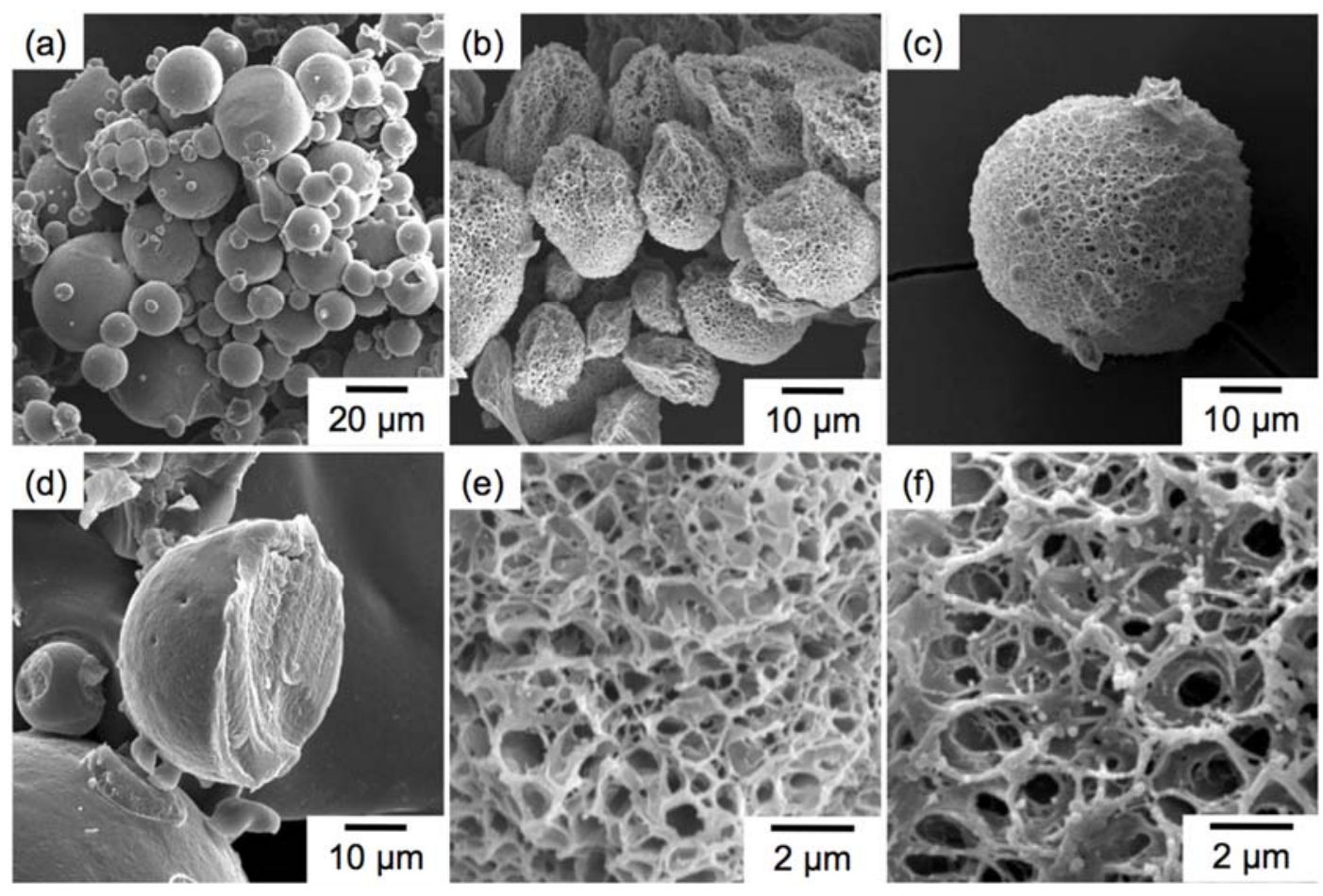

Figure 8. SEM photographs of vacuum-dried (a,d) and freeze-dried (b,c,e,f) cellulose particles prepared by SRM of cellulose-[Bmim]Cl-DMF ((a,b,d,e) 7/43/50 and (c,f) 3/47/50, w/w/w) droplets dispersed in HD with a Silaplane FM-3321 using 1-butanol, 
and then washed. Before (a) and after (d) fractioning with a razor blade. Low (b,c) and high (e,f) magnification.

\section{Conclusion}

Micrometer-sized spherical cellulose particles were successfully prepared by SRM, whereby cellulose-[Bmim]Cl-DMF droplets dispersed in HD were mixed with 1-butanol. This method is a simple approach for the preparation of cellulose particles having various structures using ionic liquid, which is different to the previous methods. $[8,41]$ The structure could be controlled by varying the drying conditions; cellulose particles with spongy and dense structures were prepared by applying and not applying the freeze-drying technique, respectively.

Interestingly, obtained spongy cellulose particles could be impregnated and replaced with solvents without deforming morphology by only changing surrounding medium regardless of the polarity. We confirmed that spongy cellulose particles could be impregnated with the monomers such as styrene and methyl methacrylate without change of particle morphology (size). Further investigation of the cellulose-polymer composite particles is in progress, in which the spongy cellulose particles are used as template utilizing the above absorption behavior. This structure is expected to 
reinforce the strength of matrix polymers such as carbon fiber composite materials.[48-50]

\section{Acknowledgment}

This research was supported by Adaptable and Seamless Technology Transfer Program through Target-driven R\&D, Japan Science and Technology Agency, Shorai Foundation for Science and Technology, and a Grant-in-Aid for Scientific Research (grant no. 23350114).

\section{References}

[1] D. Klemm, B. Heublein, H.P. Fink, A. Bohn, Angew. Chem. Int. Ed. 44 (2005) 3358.

[2] M. Nogi, S. Iwamoto, A.N. Nakagaito, H. Yano, Adv. Mater. 21 (2009) 1595.

[3] R.J. Moon, A. Martini, J. Nairn, J. Simonsen, J. Youngblood, Chem. Soc. Rev. 40 (2011) 3941.

[4] S.J. Eichhorn, Soft Matter 7 (2011) 303.

[5] C. Carrick, M. Ruda, B. Pettersson, P.T. Larsson, L. Wagberg, Rsc Advances 3 (2013) 2462.

[6] M. Gericke, J. Trygg, P. Fardim, Chem. Rev. 113 (2013) 4812.

[7] M. Ettenauer, F. Loth, K. Thummler, S. Fischer, V. Weber, D. Falkenhagen, Cellulose 18 (2011) 1257.

[8] K.F. Du, M. Yan, Q.Y. Wang, H. Song, J. Chromatogr. A 1217 (2010) 1298.

[9] A.F. Metaxa, E.K. Efthimiadou, N. Boukos, G. Kordas, J. Colloid Interface Sci. 384 (2012) 198. 
[10] W. DeOliveira, W.G. Glasser, J. Appl. Polym. Sci. 60 (1996) 63.

[11] R. Sescousse, R. Gavillon, T. Budtova, J. Mater. Sci 46 (2011) 759.

[12] S. Nagaoka, H. Tobata, Y. Takiguchi, T. Satoh, T. Sakurai, M. Takafugi, H. Ihara, J. Appl. Polym. Sci. 97 (2005) 149.

[13] D.M. Wang, G. Hao, Q.H. Shi, Y. Sun, J. Chromatogr. A 1146 (2007) 32.

[14] J.R. Correa, E. Bordallo, D. Canetti, V. Leon, L.C. Otero-Diaz, C. Negro, A. Gomez, R. Saez-Puche, Mater. Res. Bull. 45 (2010) 946.

[15] Y.L. Guan, X.F. Liu, Y.P. Zhang, K.D. Yao, J. Appl. Polym. Sci. 67 (1998) 1965.

[16] J. Cai, S. Kimura, M. Wada, S. Kuga, L. Zhang, Chemsuschem 1 (2008) 149.

[17] C.W. Kim, D.S. Kim, S.Y. Kang, M. Marquez, Y.L. Joo, Polymer 47 (2006) 5097.

[18] D.M. Phillips, L.F. Drummy, D.G. Conrady, D.M. Fox, R.R. Naik, M.O. Stone, P.C. Trulove, H.C. De Long, R.A. Mantz, J. Am. Chem. Soc. 126 (2004) 14350.

[19] H.B. Xie, S.B. Zhang, S.H. Li, Green Chem. 8 (2006) 630.

[20] K. Kinoshita, H. Minami, Y. Tarutani, M. Okubo, Chem. Lett. 39 (2010) 741.

[21] K. Kinoshita, H. Minami, Y. Tarutani, K. Tajima, M. Okubo, H. Yanagimoto, Langmuir 27 (2011) 4474.

[22] R.P. Swatloski, S.K. Spear, J.D. Holbrey, R.D. Rogers, J. Am. Chem. Soc. 124 (2002) 4974 .

[23] A. Pinkert, K.N. Marsh, S.S. Pang, M.P. Staiger, Chem. Rev. 109 (2009) 6712.

[24] H. Wang, G. Gurau, R.D. Rogers, Chem. Soc. Rev. 41 (2012) 1519.

[25] A.R. Xu, J.J. Wang, H.Y. Wang, Green Chem. 12 (2010) 268.

[26] N. Luo, Y.X. Lv, D.X. Wang, J.M. Zhang, J. Wu, J.S. He, J. Zhang, Chem. Commun. 48 (2012) 6283.

[27] R. Rinaldi, Chem. Commun. 47 (2011) 511.

[28] M. Gericke, T. Liebert, O.A. El Seoud, T. Heinze, Macromol. Mater. Eng. 296 (2011) 483.

[29] M.B. Turner, S.K. Spear, J.D. Holbrey, R.D. Rogers, Biomacromolecules 5 (2004) 1379.

[30] L. Hardelin, J. Thunberg, E. Perzon, G. Westman, P. Walkenstrom, P. Gatenholm, J. Appl. Polym. Sci. 125 (2012) 1901.

[31] M.G. Freire, A.R.R. Teles, R.A.S. Ferreira, L.D. Carlos, J.A. Lopes-da-Silva, J.A.P. Coutinho, Green Chem. 13 (2011) 3173.

[32] A. Takegawa, M. Murakami, Y. Kaneko, J. Kadokawa, Carbohydr. Polym. 79 (2010) 85.

[33] N. Hameed, Q.P. Guo, F.H. Tay, S.G. Kazarian, Carbohydr. Polym. 86 (2011) 94.

[34] A. Takegawa, M. Murakami, Y. Kaneko, J. Kadokawa, Polym. Compos. 30 (2009) 
1837.

[35] J.I. Kadokawa, M.A. Murakami, Y. Kaneko, Carbohydr. Res. 343 (2008) 769.

[36] T. Meng, X. Gao, J. Zhang, J.Y. Yuan, Y.Z. Zhang, J.S. He, Polymer 50 (2009) 447.

[37] S. Kohler, T. Liebert, T. Heinze, J. Polym. Sci., Part A: Polym. Chem. 46 (2008) 4070 .

[38] L. Feng, Z.I. Chen, J. Mol. Liq. 142 (2008) 1.

[39] J. Wu, J. Zhang, H. Zhang, J.S. He, Q. Ren, M. Guo, Biomacromolecules 5 (2004)

266.

[40] K. Nakashima, K. Yamaguchi, N. Taniguchi, S. Arai, R. Yamada, S. Katahira, N. Ishida, H. Takahashi, C. Ogino, A. Kondo, Green Chem. 13 (2011) 2948.

[41] F. Shi, D.Q. Lin, W. Phottraithip, S.J. Yao, J. Appl. Polym. Sci. 119 (2011) 3453.

[42] M. Okubo, Y. Konishi, S. Sebki, H. Minami, Colloid. Polym. Sci. 280 (2002) 765.

[43] M. Okubo, Y. Konishi, M. Takebe, H. Minami, Colloid. Polym. Sci. 278 (2000) 919.

[44] T. Tanaka, M. Okayama, H. Minami, M. Okubo, Langmuir 26 (2010) 11732.

[45] A. Kumar, Y.S. Negi, N.K. Bhardwaj, V. Choudhary, Carbohydr. Polym. 88 (2012) 1364 .

[46] H.S. Qi, T. Liebert, T. Heinze, Cellulose 19 (2012) 925.

[47] M. Tabuchi, Nat. Biotechnol. 25 (2007) 389.

[48] A. Ben Mabrouk, M.R. Vilar, A. Magnin, M.N. Belgacem, S. Boufi, J. Colloid Interface Sci. 363 (2011) 129.

[49] N. Isobe, M. Sekine, S. Kimura, M. Wada, S. Kuga, Cellulose 18 (2011) 327.

[50] T. Abitbol, T. Johnstone, T.M. Quinn, D.G. Gray, Soft Matter 7 (2011) 2373. 
Graphical Abstract
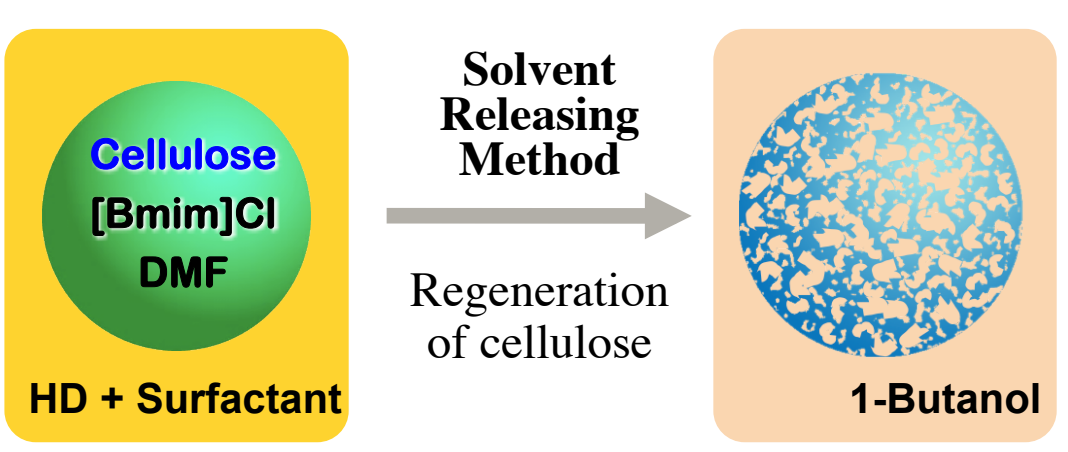

Vacuum -drying

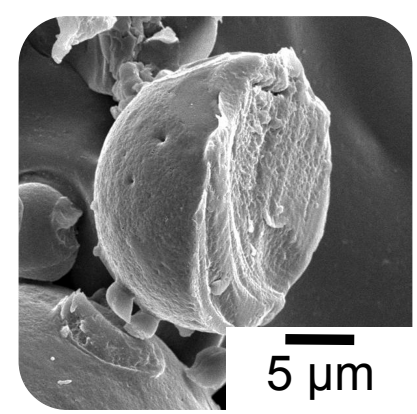

Cellulose particle

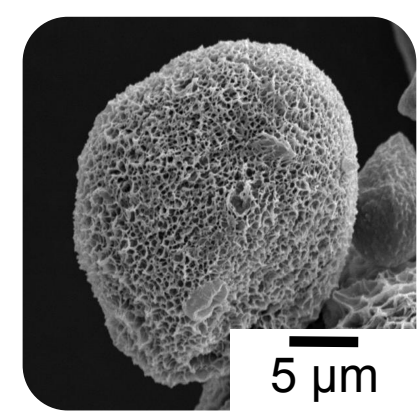

